\title{
Fecal Immunochemical Test and Fecal Calprotectin Results Show Different Profiles in Disease Monitoring for Ulcerative Colitis
}

\author{
Sakiko Hiraoka ${ }^{1}$, Toshihiro Inokuchi ${ }^{1}$, Asuka Nakarai ${ }^{1}$, Shiho Takashima ${ }^{1}$, Daisuke Takei $^{1}$, Yuusaku Sugihara ${ }^{1}$, Masahiro \\ Takahara $^{1}$, Keita Harada ${ }^{2}$, Hiroyuki Okada ${ }^{1}$, and Jun Kato ${ }^{3}$ \\ Departments of ${ }^{1}$ Gastroenterology and Hepatology, ${ }^{2}$ Endoscopy, Okayama University Graduate School of Medicine, Dentistry and Pharmaceutical \\ Sciences, Okayama, and ${ }^{3}$ Second Department of Internal Medicine, Wakayama Medical University, Wakayama, Japan
}

\section{See editorial on page 117.}

Background/Aims: Both fecal immunochemical test (FIT) and fecal calprotectin (Fcal) results are useful biomarkers for ulcerative colitis (UC). However, the situations in which each marker should be used are largely unknown. Methods: A total of 110 colonoscopy intervals of UC patients were assessed, and correlations between changes in colonoscopic findings and changes in the two aforementioned fecal markers were examined. Results: Among patients with mucosal healing $(\mathrm{MH})$ and negative FIT or Fcal results at the initial colonoscopy, FIT and Fcal findings exhibited accuracies of 93\% (38/41) and 79\% (26/33), respectively, for predicting the results of the subsequent examination. Among the 24 patients who showed endoscopic activity at the precedent colonoscopy and $\mathrm{MH}$ at the subsequent examination, positive-to-negative conversion of FIT and Fcal findings at the subsequent examination was observed in $92 \%(12 / 13)$ and $62 \%(8 / 13)$ of patients, respectively. Among the 43 patients who showed endoscopic activity at both the precedent and subsequent examinations, Fcal findings reflected the change in endoscopic activity better than FIT results $(r=0.59$, $p<0.0001$ vs $r=0.30, p=0.054)$. Conclusions: The FIT is useful for confirming $\mathrm{MH}$ and the occurrence of relapse. In contrast, Fcal is useful for monitoring the mucosal status of patients with active inflammation. (Gut Liver 2018;12:142148)

Key Words: Colitis, ulcerative; Quantitative fecal immunochemical test; Fecal calprotectin

\section{INTRODUCTION}

Ulcerative colitis (UC) is an idiopathic chronic inflammatory disorder that is characterized by manifestations such as diarrhea, rectal bleeding, abdominal pain, fever, anemia and body weight loss. ${ }^{1}$ Evaluating the disease activity as precisely as possible is important for achieving for better disease control. The endoscopic findings of the colonic mucosa have recently become essential in the evaluation of disease activity. The achievement of mucosal healing $(\mathrm{MH})$ has been recommended as the treatment goal of UC, because it is associated with a reduced risk of relapse and colectomy. ${ }^{2}$ However, a major disadvantage of using the mucosal status to evaluate disease activity is that it necessitates colonoscopy, which is an invasive and costly procedure. Thus, surrogate markers that allow for the evaluation of the mucosal status have been sought. Among several candidates, fecal calprotectin (Fcal) has become a front-runner, particularly in Western countries. ${ }^{3,4}$ Meanwhile, we have reported the usefulness of the fecal immunochemical test (FIT) in evaluating the mucosal status and predicting MH in UC. ${ }^{5-7}$

The Fcal and FIT results have both been shown to be closely correlated to the endoscopic activity in UC patients, ${ }^{3,7-14}$ and flare-ups in UC patients can be efficiently predicted by monitoring either marker. ${ }^{15-18}$ Although FIT has advantages over an Fcal analysis in terms of the cost of measurement (which is much lower) and in its sensitivity for predicting $\mathrm{MH}^{5-7}$ particularly complete MH (defined by a Mayo endoscopic subscore [MES] of 0 alone), there have been fewer reports showing the performance of FIT in UC. Thus, it remains unclear which of these markers should be applied in clinical practice. In this regard, there may be clinical scenarios in which FIT or Fcal is more effective.

The aim of the present study was to determine the best choice

Correspondence to: Sakiko Hiraoka

Department of Gastroenterology and Hepatology, Okayama University Graduate School of Medicine, Dentistry and Pharmaceutical Sciences, 2-5-1 Shikata-cho, Kita-ku, Okayama 700-8558, Japan

Tel: +81-86-235-7219, Fax: +81-86-225-5991, E-mail: sakikoh@cc.okayama-u.ac.jp Received on January 5, 2017. Revised on April 1, 2017. Accepted on April 19, 2017. Published online September 7, 2017 pISSN 1976-2283 eISSN 2005-1212 https://doi.org/10.5009/gnl17013

() This is an Open Access article distributed under the terms of the Creative Commons Attribution Non-Commercial License (http://creativecommons.org/licenses/by-nc/4.0) which permits unrestricted non-commercial use, distribution, and reproduction in any medium, provided the original work is properly cited. 
of fecal markers in real clinical situations, by evaluating the changes in the values of each marker according to the changes in the endoscopic findings of UC patients.

\section{MATERIALS AND METHODS}

\section{Patients}

Since March 2013, we have requested that UC patients who are scheduled to undergo colonoscopy at Okayama University Hospital submit two fecal samples for FIT and Fcal examinations on the day of colonoscopy. In this study, the patients who underwent two or more colonoscopies and who also provided fecal samples between March 2013 and December 2015 were consecutively identified, and the correlations between the changes of the colonoscopic findings and the changes in the fecal markers were analyzed between two colonoscopies. In the patients who underwent three colonoscopies during the study period, two adjacent colonoscopies were compared. Similarly, in those with four colonoscopies, three comparisons were made.

This study was approved by the Institutional Review Board of Okayama University Graduate School of Medicine (IRB number: 1611-015). Informed consent was obtained from each patient.

\section{Fecal sampling and the FIT analysis}

The details of the method used for the FIT analysis have been described previously. ${ }^{5}$ Briefly, the patients prepared fecal samples within 2 days before colonoscopy using an OCHemodia sampling probe (Eiken Chemical, Tokyo, Japan), which was provided by the manufacturer. The patient inserts the probe into several different areas of stool and then firmly places it back into the tube to seal it. The probe tip with the fecal sample is suspended in a standard volume of hemoglobin-stabilizing buffer. The submitted stool samples were immediately processed and examined using an OC-Sensor neo or DIANA (Eiken Chemical) system, which can accurately measure fecal hemoglobin at concentrations of 50 to $1,000 \mathrm{ng} / \mathrm{mL}$. Fecal specimens with a hemoglobin concentration of $>1,000 \mathrm{ng} / \mathrm{mL}$ were measured following dilution. Because FIT is not accurate for measuring hemoglobin concentrations of $<50 \mathrm{ng} / \mathrm{mL}$, the specimens with a hemoglobin concentration within this range were categorized as one (0 to $50 \mathrm{ng} / \mathrm{mL}$ ). Based on our previous reports, ${ }^{5,6,19}$ a negative FIT result was defined by a fecal hemoglobin concentration of $<100 \mathrm{ng} / \mathrm{mL}$, and a positive FIT result was defined by a concentration of $>100 \mathrm{ng} / \mathrm{mL}$.

\section{The Fcal analysis}

The fecal samples collected by the patients were stored at $-70^{\circ} \mathrm{C}$ until shipment to the laboratory where the calprotectin analysis was performed. The samples were sent to the Institute of Applied Technology for Innate Immunity (Kagawa, Japan), where the level of calprotectin in the stool specimens was measured using a PhiCal ${ }^{\circledR}$ Calprotectin ELISA kit (Immundiagnostik
AG, Bensheim, Germany). The quantitative range was 0.65 to $84,000 \mu \mathrm{g} / \mathrm{g}$ after the appropriate dilution of fecal samples (to 1:50-1:100,000). Because the cutoff values for Fcal varied widely among the different published studies, the cutoff value in this study was set at $180 \mu \mathrm{g} / \mathrm{g}$ based on the results of a receiver operating characteristic curve analysis (Supplementary Fig. 1).

\section{Colonoscopy}

Bowel preparation was performed with a polyethylene glycolbased or magnesium citrate-based electrolyte solution according to the standard protocol of our hospital. After the colonic lavage fluid was cleared, the patients underwent colonoscopy. Procedures were excluded from the study if the colonoscopic examination was incomplete due to problems with the bowel preparation or if the colonoscope could not be inserted into the cecum.

The mucosal status of the UC patients was assessed according to the MES classification. ${ }^{20}$ The evaluation was performed at each portion of the colorectum (the cecum and ascending colon combined, the transverse colon, descending colon, sigmoid colon, and rectum), and the maximum score in the colorectum of each patient was used for the analysis. $\mathrm{MH}$ was defined as an MES of 0 throughout the colorectum. All of the colonoscopic examinations were performed by experienced colonoscopists who were blinded to the FIT and Fcal results. The final determination of the MES of each patient was made by independent investigators who were not of aware of the patient's status or symptoms.

\section{Statistical analysis}

Statistical analyses were conducted using the JMP version 12.0 for Windows (SAS Institute Inc., Cary, NC, USA). The sensitivity, specificity, positive predictive value (PPV) and negative predictive value (NPV) with 95\% confidence intervals (CIs) for detecting the mucosal status were determined based on the FIT and Fcal results. Spearman rank correlation test was performed to determine the correlation coefficient between the change of the fecal marker values and the change of the MES. All p-values were two-sided. The $p$-values of $<0.05$ were considered to indicate statistical significance.

\section{RESULTS}

\section{The clinical characteristics of the patients}

During the study period, 84 UC patients underwent two or more colonoscopies. Of these, 62 patients received two colonoscopies (one interval each), 18 received three colonoscopies (two intervals each), and four patients received four colonoscopies (three intervals each) during the study period. Thus, a total of 110 intervals between adjacent colonoscopic examinations were identified. Table 1 summarizes the characteristics of the study patients and the colonoscopy findings. Forty-nine (58\%) of the 
84 UC patients were men and the median age at the onset of UC was 33 years. Fifty-seven patients (68\%) had pancolitis, 22 (26\%) had left-side colitis, and five (6\%) had proctitis. The median disease duration at the precedent colonoscopy was 122 months (range, 72 to 516 months). The interval between the two adjacent colonoscopic examinations that were compared was 13.3 months (range, 1.9 to 32.0 months). The colonoscopy findings

Table 1. Characteristics of the Study Patients and the Colonoscopy Findings

\begin{tabular}{lc}
\hline \multicolumn{1}{c}{ Variable } & Value \\
\hline Patients & 84 \\
Total & $33(9-77)$ \\
Age at onset, yr & $122(72-516)$ \\
Duration of disease at precedent colonoscopy, mo & \\
Sex & 49 \\
$\quad$ Male & 35 \\
$\quad$ Female & \\
Extent of disease & 57 \\
$\quad$ Pancolitis & 22 \\
$\quad$ Left-side colitis & 5 \\
$\quad$ Proctitis & \\
Analyzed colonoscopic intervals & $62 / 18 / 4$ \\
1/2/3 & \\
Colonoscopies & 110 \\
Total & $13.3(1.9-32.0)$ \\
Interval between the precedent and subsequent \\
colonoscopic examinations, mo \\
Colonoscopy findings \\
$\quad$ MESo/ MES1/MES2/MES3 \\
\hline
\end{tabular}

Data are presented as number or median (range).

MES, Mayo endoscopic subscore.

A

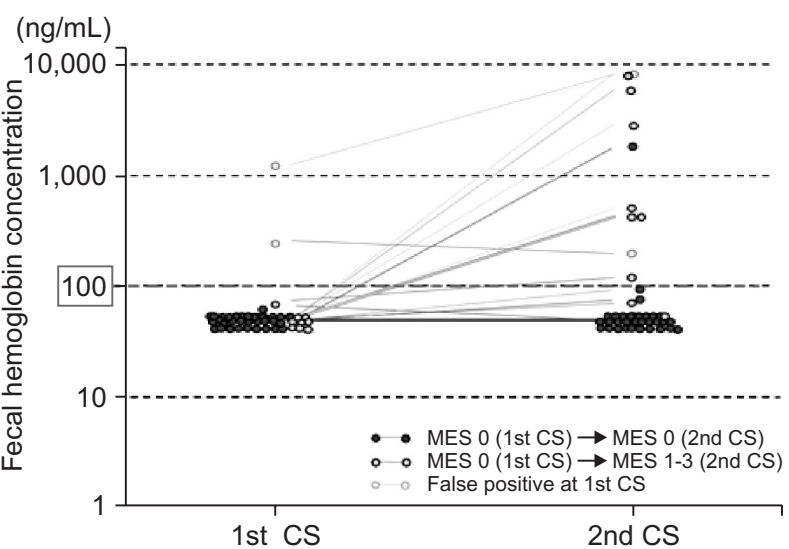

revealed that the maximum MES for the colorectum at the precedent examination was MES 0 in 43 cases (39\%), MES 1 in 32 cases (29\%), MES 2 in 29 cases (26\%), and MES 3 in six cases (5\%).

\section{The changes in the endoscopic findings between the two adjacent colonoscopic examinations}

The changes in the endoscopic activity in 110 intervals from the precedent colonoscopic examination to the subsequent examination are shown in Fig. 1. At the precedent examination, 43 patients showed MH (MES 0), and the remaining 67 had endoscopic activity (MES 1-3). Thirty-two of the 43 (74\%) cases with MES 0 also had MES 0 at the subsequent examination, while 11 of the 43 cases (26\%) developed endoscopic activity (MES 1-3). On the other hand, among the 67 cases with MES 1-3 at the precedent examination, 24 (36\%) achieved MH (MES 0 ) at the subsequent examination, while the remaining 43 (64\%) continued to present endoscopic activity. Among the 43 patients with sustained endoscopic activity during the interval of the two examinations, 12 showed a decreased MES value, 22 maintained the same level activity, and nine presented an increased

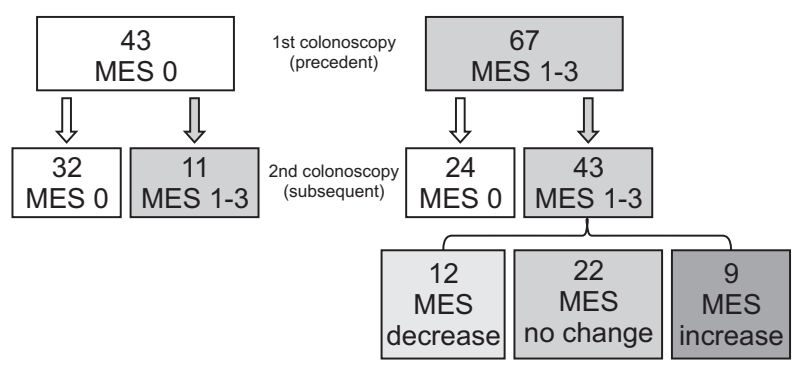

Fig. 1. Changes in mucosal activity between two consecutive colonoscopies.

MES, Mayo endoscopic subscore.

B

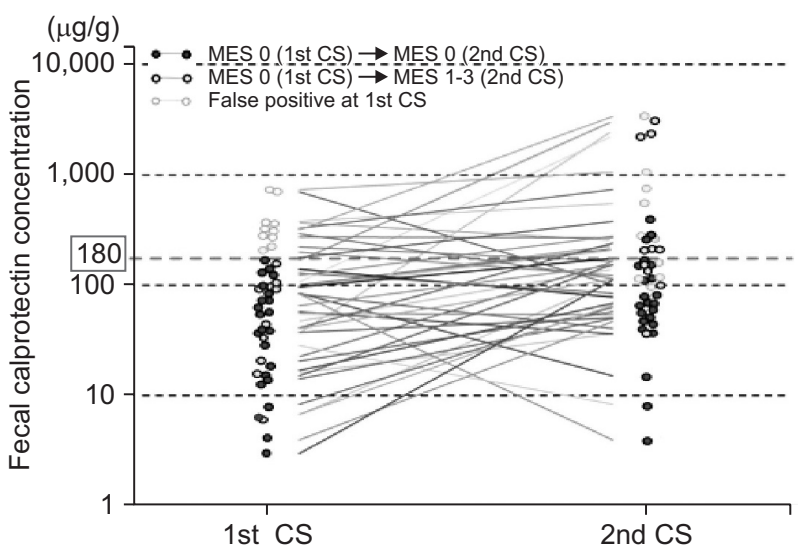

Fig. 2. Changes in fecal marker values in patients with mucosal healing at the precedent colonoscopy. (A) Fecal immunochemical test and (B) fecal calprotectin. MES, Mayo endoscopic subscore; 1st CS, precedent colonoscopy; 2nd CS, subsequent CS. 
MES value.

\section{The changes in the fecal marker levels in the patients with MH at the precedent colonoscopic examination}

Based on the data from all 110 precedent colonoscopies, the sensitivity of FIT for predicting MESO was higher than that of Fcal (0.95 [95\% CI, 0.89 to 1.02] vs 0.77 [95\% CI, 0.64 to 0.89]), and the specificity of the two markers was similar (0.62 [95\% CI, 0.50 to 0.74 ] vs 0.67 [95\% CI, 0.56 to 0.78$]$ ]). Fig. 2 shows the changes in the fecal marker levels of each of the 43 cases with $\mathrm{MH}$ at the precedent colonoscopic examination. Of the 32 patients who exhibited $\mathrm{MH}$ at both the precedent and subsequent examinations, 30 (94\%) and 29 (91\%) were negative on the FIT at the precedent and subsequent examinations, respectively. On the other hand, 22 (69\%) and 21 (66\%) were negative on the Fcal analysis at the precedent and subsequent examinations, respectively. Among the 11 patients who showed MH at the precedent examination but active inflammation at the subsequent examination, all $11(100 \%)$ had a negative FIT result at the precedent examination and nine (82\%) had a positive FIT result at the subsequent examination. In contrast, among these, 10 (91\%) had a negative Fcal result at the precedent examination, and only six (60\%) had a positive Fcal result at the subsequent examination.

The overall accuracy of FIT in predicting the results of the subsequent colonoscopic examination in patients who showed MH with a negative FIT result at the precedent examination was 93\% (38/41), while the accuracy of the Fcal analysis in the patients who showed $\mathrm{MH}$ with a negative Fcal result at the precedent examination was 79\% (26/33). These results suggest that the FIT is superior to the Fcal analysis for predicting $\mathrm{MH}$ (MES 0). In addition, the FIT results were more likely to reflect the change in the mucosal status of the patients in whom $\mathrm{MH}$ had been successfully predicted based on a negative FIT result.

\section{The changes in the fecal markers in patients with mucosal inflammation at the precedent colonoscopic examination}

Regarding the predictability of the presence of endoscopic inflammation (MES 1-3), the sensitivity was similar (0.64 [95\% CI, 0.53 to 0.76] vs 0.67 [95\% CI, 0.56 to 0.78 ] between FIT and Fcal, while the specificity was more than 10 points higher than that of Fcal (0.95 [95\% CI, 0.89 to 1.02] vs 0.77 [95\% CI, 0.64 to 0.89]), according to the results of precedent colonoscopy. In addition, the sensitivity and specificity of FIT and Fcal for presence of endoscopic inflammation based on the results of both precedent and subsequent colonoscopy were similar to above (Supplementary Table 1). Among the 24 patients who showed endoscopic activity at the precedent examination and $\mathrm{MH}$ at the subsequent examination, 13 (54\%) presented positive FIT and another subset of 13 (54\%) presented positive Fcal values at the precedent examination. Among the patients with positive FIT and those with positive Fcal values, the ratio of negative conversion of each fecal marker (consistent with achieving $\mathrm{MH}$ on endoscopy) was 92\% (12/13) and 62\% (8/13), respectively (Fig. 3). These results suggest that neither of the markers is particularly useful for predicting active inflammation, and that FIT would be better for predicting the achievement of $\mathrm{MH}$ in patients who had previously shown endoscopic activity.

Finally, we analyzed the difference in the performance of the two fecal markers in patients who showed endoscopic activity at both the precedent and subsequent colonoscopic examinations. Fig. 4 shows the relationship between the changes in the fecal makers and the changes in the endoscopic activity between the precedent and subsequent examinations. Among these subjects, Fcal reflected the change of endoscopic activity better than FIT $(r=0.59, p<0.0001$ vs $r=0.30, p=0.054)$. This suggests that Fcal
A

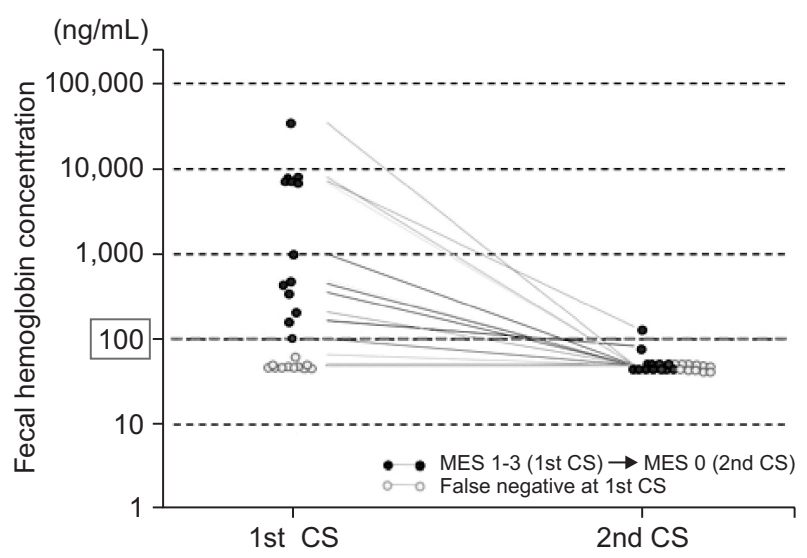

B

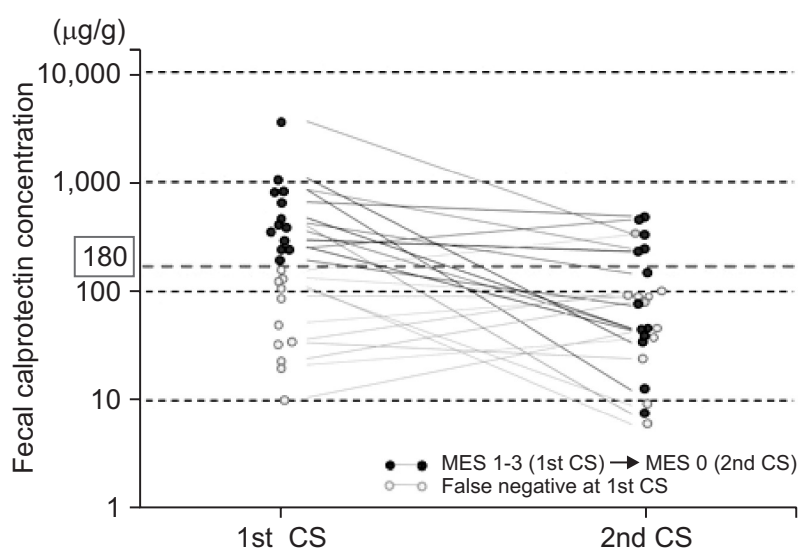

Fig. 3. Changes in fecal marker values in patients who exhibited active inflammation at the precedent colonoscopy and mucosal healing at the subsequent colonoscopy. (A) Fecal immunochemical test and (B) fecal calprotectin. 1st CS, precedent colonoscopy; 2nd CS, subsequent CS; MES, Mayo endoscopic subscore. 


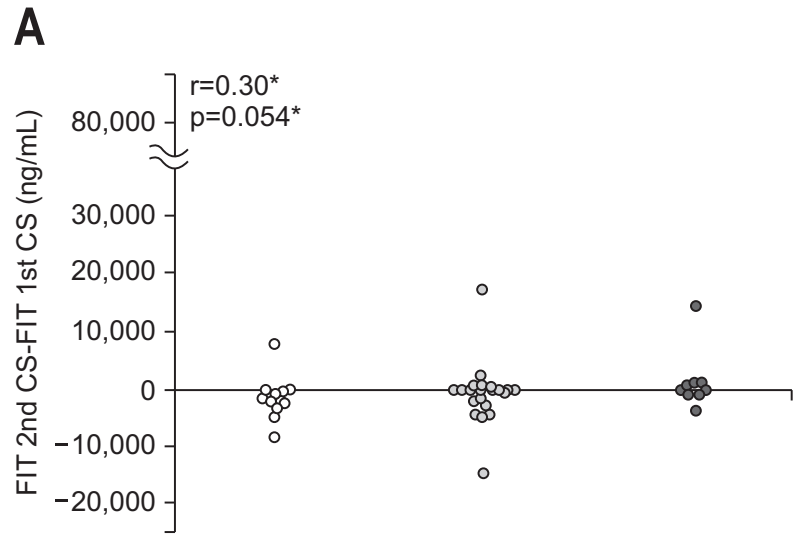

MES decrease MES no change MES increase
B

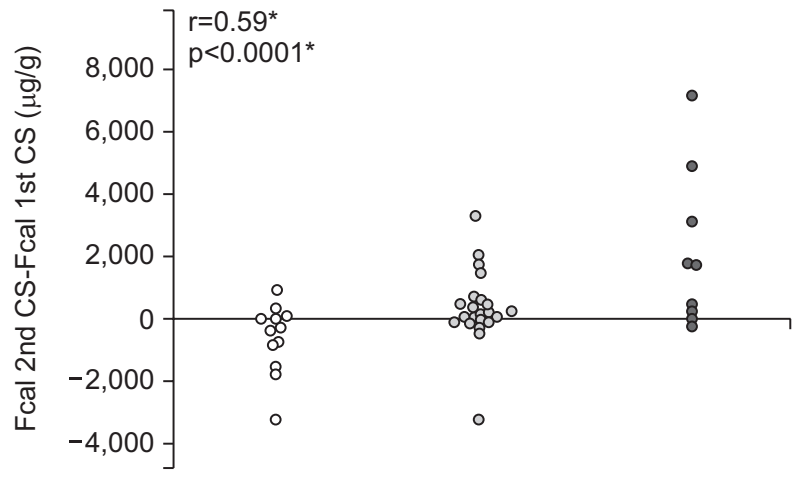

MES decrease MES no change MES increase

Fig. 4. Differences in fecal marker values between precedent and subsequent colonoscopies according to the change in the degree of inflammation. This analysis was performed for 43 patients who exhibited active inflammation in both colonoscopies. (A) Fecal immunochemical test and (B) fecal calprotectin.

FIT, fecal immunochemical test; 1st CS, precedent colonoscopy; 2nd CS, subsequent CS; MES, Mayo endoscopic subscore; Fcal, fecal calprotectin. *Spearman rank correlation test.

would be superior to FIT for monitoring endoscopic activity in patients with persistent UC activity.

\section{DISCUSSION}

In the present study, we evaluated the difference in the performance of the Fcal analysis and the FIT in evaluating UC patients in the clinical setting, by comparing the changes in each marker with the changes in endoscopic activity. We have found that the FIT appears to be superior to the Fcal analysis for predicting MH (MES 0) both in the previous and current reports. Distinct from the results of our previous paper, the highlight of this study was that we have clearly presented the usefulness of fecal markers for disease monitoring and which marker should be applied at specific clinical situations of disease courses of UC. The FIT was more likely to reflect the changes in the mucosal status of patients in whom the fecal marker had successfully predicted MH. In contrast, the Fcal analysis was superior to the FIT for monitoring the changes in endoscopic activity in patients with persistent UC activity.

Fcal, a calcium-binding heterodimer which is abundant in cytoplasm of neutrophil, is the most widely used surrogate marker of mucosal inflammation. Fcal has been shown to predict the presence of active inflammation in UC patients with sensitivity ranging from $71 \%$ to $93 \%$ and specificity ranging from $71 \%$ to $100 \%{ }^{3,4,7}$ This fecal protein has also been used as a marker of $\mathrm{MH}$, and the sensitivity and specificity for predicting $\mathrm{MH}$ has been reported to be $65 \%$ to $100 \%$, and $53 \%$ to $91 \%$, respectively. ${ }^{6,9-14}$

In the present study, the sensitivity of Fcal for detecting the presence of endoscopic activity (67\%) and MH (77\%) was lower in comparison to previous studies. The major reason for the lower performance was probably the difference in the definition of MH (MES 0 alone) and active inflammation (MES 1-3). Until now, the definition of MH has not been established. Older reports were likely to define MH as MES 0 or $1,{ }^{2,21}$ whereas more recent studies have defined $\mathrm{MH}$ as MES 0 alone. ${ }^{13,14,22,23}$ We previously observed that the risk of relapse differed significantly between patients with MES 0 and those with MES $1 .^{22}$ Although further studies are required to confirm the difference in the prognosis between the definitions of $\mathrm{MH}$, the higher sensitivity of FIT in comparison to Fcal in predicting MH (MES 0 alone) suggests that FIT might be more useful than Fcal for predicting a reduced risk of relapse.

We determined the cutoff (180 $\mu \mathrm{g} / \mathrm{g})$ in this study based on receiver operating characteristics curve analysis, because the optimal cutoff of Fcal have not yet been established. Previous studies have reported that the sensitivity and specificity of Fcal for predicting MES 0 by the cutoffs 100 to $200 \mu \mathrm{g} / \mathrm{g}$ were $65 \%$ to $77 \%$ and $53 \%$ to $91 \%$, respectively. ${ }^{6,10,13,14}$ And our results (77\% sensitivity and 67\% specificity) were consistent with those previous results. To further verify the appropriateness of this cutoff, alternative cutoffs (100 to $140 \mu \mathrm{g} / \mathrm{g}$ ) were also examined. However, the lower cutoffs did not prove to be superior to the cutoff $180 \mu \mathrm{g} / \mathrm{g}$ with regard to predictability for MH (data not shown).

FIT enabled for assessment of the fecal hemoglobin concentrations using an antibody specific for human hemoglobin, and has proven to be the available noninvasive test for screen for colorectal neoplasia. ${ }^{24}$ We previously reported that FIT can predict $\mathrm{MH}$ (MES 0) with 92\% to 95\% sensitivity and 62\% to $71 \%$ specificity; $;^{5,6}$ the values of the present study are in line with our previous findings. In contrast, the sensitivity of a positive FIT result for predicting the presence of endoscopic activity (MES $1-3)$ in this study was relatively low (0.64), which suggests that a considerable number of the subjects with slight inflammation 


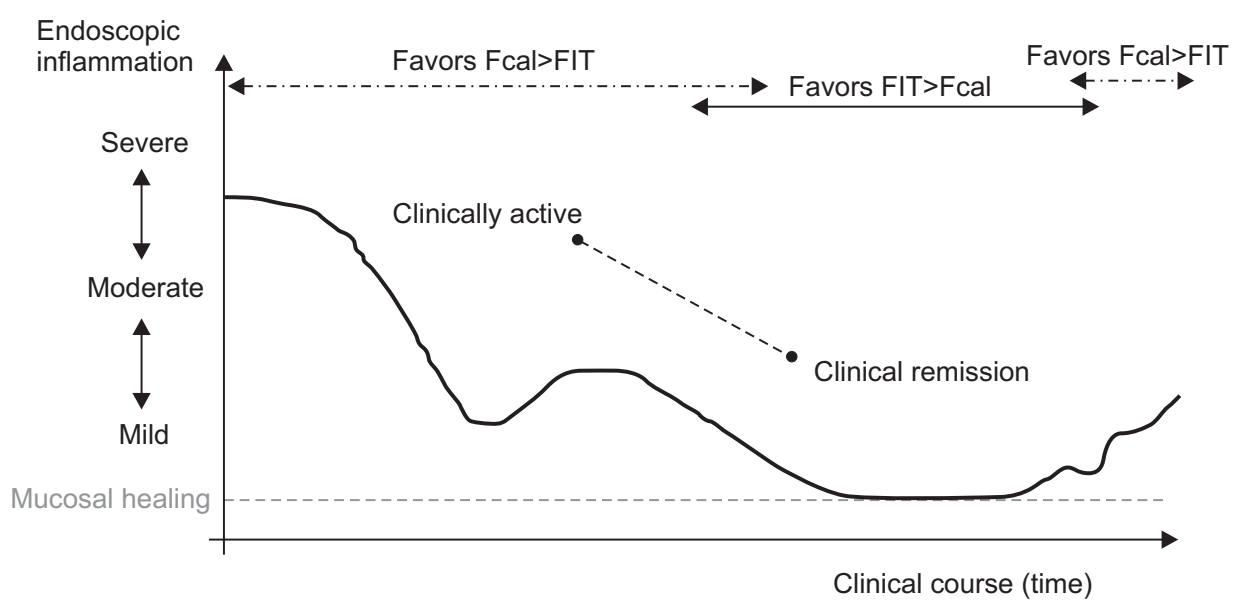

Fig. 5. The proposed optimal usage of fecal markers during the clinical course of ulcerative colitis.

FIT, fecal immunochemical test; Fcal, fecal calprotectin.
(MES 1) showed false-negative results. In this regard, FIT appears to be sensitive to a lack of inflammation rather than presence of inflammation. MH, particularly MES 0 alone, is a very rigorous endpoint for potential biomarkers of UC, and the stable results in predicting $\mathrm{MH}$ suggest the robustness of the marker. In this regard, FIT and the cutoff values for predicting $\mathrm{MH}$ are robust, as has been shown in our previous reports, ${ }^{5,6,19}$ and the superiority in predicting the changes in relation to $\mathrm{MH}$ that were observed in the present study might be attributable to this characteristic of FIT.

In association with the higher ability to predict the mucosal changes associated with $\mathrm{MH}$, our previous study demonstrated that none of the patients who maintained a negative FIT result experienced clinical relapse during 2.5 years of follow-up. On the other hand, 63\% patients who showed positive conversion in their FIT results during the study period experienced relapse. ${ }^{19}$ Thus, FIT appears to be particularly useful in predicting the achievement of $\mathrm{MH}$ in patients with active inflammation who are receiving remission induction therapy as well as patients in clinical remission who are receiving maintenance therapy (conversion from positive to negative). In addition, FIT would be helpful for predicting relapse because the marker is sensitive to the development of inflammation from $\mathrm{MH}$ (conversion from negative to positive).

On the other hand, as shown in Fig. 4, the Fcal analysis was more sensitive than the FIT to the changes in endoscopic activity in patients who had persistent UC activity. Thus, in the clinical setting, the Fcal analysis appears to be more suitable for the early monitoring of the treatment efficacy in UC patients with ongoing mucosal activity. In this clinical situation, FIT may be inaccurate because the solidification of stools along with treatment efficacy could lead to a temporary increase in bleeding.

Given the high cost of the Fcal analysis, however, its use should be minimized and the use of FIT should be maximized in clinical practice. On the basis of our results and the costeffectiveness, we propose the following protocol for the clinical management of UC using fecal markers (Fig. 5). Active UC pa- tients starting induction therapy should be monitored with Fcal to evaluate the efficacy of their treatment. After the improvement of their clinical symptoms, the patients should be monitored by FIT due to its higher PPV for MH and lower cost. After the negative conversion of FIT, endoscopy should be considered to confirm the achievement of $\mathrm{MH}$. After the achievement of $\mathrm{MH}$, FIT should be continuously used to monitor disease activity. Fcal should be used after positive conversion is observed on an FIT, and colonoscopy should be appropriately timed to decide whether additional therapy should be administered.

The present study is associated with some limitations. In particular, common drawbacks are inevitably present due to the relatively small number of patients and the retrospective nature of the design. However, the retrospective design does not appear to have reduced the value of this study largely, because the actual analysis was made in a cross-sectional manner. Prospective studies on this type of topic would be cumbersome to perform and costly due to the frequent need for colonoscopy and fecal examinations.

In conclusion, FIT has an advantage in that negative results can effectively predict MH. In real clinical practice, the marker would particularly be useful to confirm the achievement and maintenance of $\mathrm{MH}$ and in predicting a relapse. On the other hand, Fcal is beneficial for monitoring the mucosal status of patients with active inflammation, indicating that the marker has strength in evaluating the efficacy of treatment, particularly in the early period after the initiation of treatment. The optimal use of the two fecal markers would lead UC patients toward better medical care.

\section{CONFLICTS OF INTEREST}

No potential conflict of interest relevant to this article was reported. 


\section{REFERENCES}

1. Podolsky DK. Inflammatory bowel disease. N Engl J Med 2002;347:417-429.

2. Peyrin-Biroulet L, Ferrante M, Magro F, et al. Results from the 2nd Scientific Workshop of the ECCO. I: impact of mucosal healing on the course of inflammatory bowel disease. J Crohns Colitis 2011;5:477-483.

3. Schoepfer AM, Beglinger C, Straumann A, Trummler M, Renzulli P, Seibold F. Ulcerative colitis: correlation of the Rachmilewitz endoscopic activity index with fecal calprotectin, clinical activity, C-reactive protein, and blood leukocytes. Inflamm Bowel Dis 2009;15:1851-1858

4. D'Haens G, Ferrante M, Vermeire S, et al. Fecal calprotectin is a surrogate marker for endoscopic lesions in inflammatory bowel disease. Inflamm Bowel Dis 2012;18:2218-2224.

5. Nakarai A, Kato J, Hiraoka S, et al. Evaluation of mucosal healing of ulcerative colitis by a quantitative fecal immunochemical test. Am J Gastroenterol 2013;108:83-89.

6. Takashima S, Kato J, Hiraoka S, et al. Evaluation of mucosal healing in ulcerative colitis by fecal calprotectin vs. fecal immunochemical test. Am J Gastroenterol 2015;110:873-880.

7. Kato J, Hiraoka S, Nakarai A, Takashima S, Inokuchi T, Ichinose M. Fecal immunochemical test as a biomarker for inflammatory bowel diseases: can it rival fecal calprotectin? Intest Res 2016;14:5-14.

8. Schoepfer AM, Beglinger C, Straumann A, et al. Fecal calprotectin more accurately reflects endoscopic activity of ulcerative colitis than the Lichtiger Index, C-reactive protein, platelets, hemoglobin, and blood leukocytes. Inflamm Bowel Dis 2013;19:332-341.

9. Mooiweer E, Fidder HH, Siersema PD, Laheij RJ, Oldenburg B. Fecal hemoglobin and calprotectin are equally effective in identifying patients with inflammatory bowel disease with active endoscopic inflammation. Inflamm Bowel Dis 2014;20:307-314.

10. Lobatón T, Rodríguez-Moranta F, Lopez A, Sánchez E, RodríguezAlonso L, Guardiola J. A new rapid quantitative test for fecal calprotectin predicts endoscopic activity in ulcerative colitis. Inflamm Bowel Dis 2013;19:1034-1042.

11. Nancey S, Boschetti G, Moussata D, et al. Neopterin is a novel reliable fecal marker as accurate as calprotectin for predicting endoscopic disease activity in patients with inflammatory bowel diseases. Inflamm Bowel Dis 2013;19:1043-1052.

12. D’Incà R, Dal Pont E, Di Leo V, et al. Calprotectin and lactoferrin in the assessment of intestinal inflammation and organic disease. Int J Colorectal Dis 2007;22:429-437.

13. Zittan E, Kelly OB, Kirsch R, et al. Low Fecal calprotectin correlates with histological remission and mucosal healing in ulcerative colitis and colonic Crohn's disease. Inflamm Bowel Dis 2016;22:623630.

14. Yamaguchi S, Takeuchi Y, Arai K, et al. Fecal calprotectin is a clinically relevant biomarker of mucosal healing in patients with quiescent ulcerative colitis. J Gastroenterol Hepatol 2016;31:9398.

15. Lasson A, Simrén M, Stotzer PO, Isaksson S, Ohman L, Strid H. Fecal calprotectin levels predict the clinical course in patients with new onset of ulcerative colitis. Inflamm Bowel Dis 2013;19:576581.

16. De Vos M, Louis EJ, Jahnsen J, et al. Consecutive fecal calprotectin measurements to predict relapse in patients with ulcerative colitis receiving infliximab maintenance therapy. Inflamm Bowel Dis 2013;19:2111-2117.

17. Jauregui-Amezaga A, López-Cerón M, Aceituno M, et al. Accuracy of advanced endoscopy and fecal calprotectin for prediction of relapse in ulcerative colitis: a prospective study. Inflamm Bowel Dis 2014;20:1187-1193.

18. Yamamoto T, Shiraki M, Bamba T, Umegae S, Matsumoto K. Fecal calprotectin and lactoferrin as predictors of relapse in patients with quiescent ulcerative colitis during maintenance therapy. Int J Colorectal Dis 2014;29:485-491.

19. Hiraoka S, Kato J, Nakarai A, et al. Consecutive measurements by Faecal immunochemical test in quiescent ulcerative colitis patients can detect clinical relapse. J Crohns Colitis 2016;10:687-694.

20. Schroeder KW, Tremaine WJ, Ilstrup DM. Coated oral 5-aminosalicylic acid therapy for mildly to moderately active ulcerative colitis: a randomized study. N Engl J Med 1987;317:1625-1629.

21. Colombel JF, Rutgeerts P, Reinisch W, et al. Early mucosal healing with infliximab is associated with improved long-term clinical outcomes in ulcerative colitis. Gastroenterology 2011;141:11941201.

22. Nakarai A, Kato J, Hiraoka S, et al. Prognosis of ulcerative colitis differs between patients with complete and partial mucosal healing, which can be predicted from the platelet count. World J Gastroenterol 2014;20:18367-18374.

23. Barreiro-de Acosta M, Vallejo N, de la Iglesia D, et al. Evaluation of the risk of relapse in ulcerative colitis according to the degree of mucosal healing (Mayo 0 vs 1): a longitudinal cohort study. J Crohns Colitis 2016;10:13-19.

24. Morikawa T, Kato J, Yamaji Y, Wada R, Mitsushima T, Shiratori Y. A comparison of the immunochemical fecal occult blood test and total colonoscopy in the asymptomatic population. Gastroenterology 2005;129:422-428. 